\title{
Psychological Distress and Quality of Life in Participants Undergoing Genetic Testing for Arrhythmogenic Right Ventricular Cardiomyopathy Caused by TMEM43 p.S358L: Is It Time to Offer Population-Based Genetic Screening?
}

\author{
Cassidy Brothers $^{a}$ Holly Etchegary ${ }^{b}$ Fiona Curtis $^{c}$ Charlene Simmonds ${ }^{d}$ \\ Jim Houston $^{\mathrm{e}}$ Terry-Lynn Young ${ }^{\mathrm{e}}$ Daryl Pullman ${ }^{\mathrm{f}}$ Hensley H. Mariathas ${ }^{\mathrm{g}}$ \\ Sean Connors $^{\text {h }}$ Kathleen Hodgkinson ${ }^{\text {b, c }}$ \\ aPostgraduate Medical Education, Faculty of Medicine, Memorial University, St. John's, NL, Canada; \\ bUnit of Clinical Epidemiology, Faculty of Medicine, Memorial University, St. John's, NL, Canada; 'Provincial \\ Medical Genetics Program, Eastern Regional Health Authority, St. John's, NL, Canada; ${ }^{d}$ Health Research Unit, \\ Faculty of Medicine, Memorial University, St. John's, NL, Canada; eDiscipline of Genetics, Faculty of Medicine, \\ Memorial University, St. John's, NL, Canada; 'Centre for Bioethics, Memorial University, St. John's, NL, Canada; \\ 'Strategy for Patient-Oriented Research Unit, Faculty of Medicine, Memorial University, St. John's, NL, Canada; \\ hDivision of Cardiology, Eastern Regional Health Authority, St. John's, NL, Canada
}

\section{Keywords}

Arrhythmogenic right ventricular cardiomyopathy · Distress · Quality of life · Population-based genetic screening

\footnotetext{
Abstract

Purpose: We have identified 27 families in Newfoundland and Labrador (NL) with the founder variant TMEM43 p.S358L responsible for 1 form of arrhythmogenic right ventricular cardiomyopathy. Current screening guidelines rely solely on cascade genetic screening, which may result in unrecognized, high-risk carriers who would benefit from preemptive implantable cardioverter-defibrillator therapy. This pilot study explored the acceptability among subjects to TMEM43 p. S358L population-based genetic screening (PBGS) in this Canadian province. Methods: A prospective cohort study assessed attitudes, psychological distress, and health-related
}

quality of life (QOL) in unselected individuals who underwent genetic screening for the TMEM43 p.S358L variant. Participants ( $n=73$ ) were recruited via advertisements and completed 2 surveys at baseline, 6 months, and 1 year which measured health-related QOL (SF-36v2) and psychological distress (Impact of Events Scale). Results: No variant-positive carriers were identified. Of those screened through a telephone questionnaire, $>95 \%$ felt positive about population-genetic screening for TMEM43 p.S358L, though 68\% reported some degree of anxiety after seeing the advertisement. There were no significant changes in health-related QOL or psychological distress scores over the study period. Conclusion: Despite some initial anxiety, we show support for PBGS among research subjects who screened negative for the TMEM43 p.S358L variant in NL. These findings have implications for future PBGS programs in the province.

(C) 2021 The Author(s) Published by S. Karger AG, Basel karger@karger.com www.karger.com/phg

Karger $\stackrel{\text { ' }}{5}$ BOPEN ACCESS
(C) 2021 The Author(s)

Published by S. Karger AG, Basel

This is an Open Access article licensed under the Creative Common Attribution-NonCommercial-4.0 International License (CC BY-NC) (http://www.karger.com/Services/OpenAccessLicense), applicable to the online version of the article only. Usage and distribution for commercial purposes requires written permission. 


\section{Introduction}

Arrhythmogenic right ventricular cardiomyopathy (ARVC) is an inherited, autosomal dominant cardiomyopathy which can lead to ventricular arrhythmias and sudden cardiac death (SCD) [1]. Its prevalence in the general population is estimated to be between $1 / 2,000$ and $1 / 5,000$, but variability in presentation and insensitive clinical tests make an accurate prevalence difficult to establish [2]. One form of ARVC, caused by the TMEM43 p.S358L pathogenic variant, was discovered in Newfoundland and Labrador (NL) and demonstrates complete penetrance and a sex-influenced phenotype [3,4]. Males present with a severe form of the disease, exhibiting cardiac symptoms and SCD at a much younger age compared to females [4]. Placement of an implantable cardioverter-defibrillator (ICD) for both primary prevention (based on variant testing alone) and secondary prevention (based on variant analysis and clinical evidence of ventricular arrhythmias) confers a significant survival benefit in TMEM43 p.S358L carriers [5]. The most substantial benefit is seen in primary prevention for male carriers, where placement of an ICD leads to a survival increase of 31 years [5]. Identifying carriers through population-based genetic screening (PBGS) could improve survival not only through placement of an ICD, but through earlier counseling of safe exercise practices given the association of ventricular arrhythmias with moderate to high-intensity exercise [6]. The lifelong risk of ventricular arrhythmias and SCD highlights the importance of identifying all individuals at risk of carrying the TMEM43 p.S358L variant who would benefit from placement of a lifesaving ICD.

Due to a major founder effect in the island's population, we have identified 27 multigenerational multiplex families with the TMEM43 p.S358L pathogenic variant that predates the peopling of the island of Newfoundland [7]. New families or extended branches of known families are often ascertained following the death of a young relative, followed by cascade genetic screening to identify gene carriers [8]. Cascade genetic screening works well within families, where the only requirement for testing is relatedness to the proband (often a deceased relative) irrespective of clinical symptoms. However, identifying new ARVC families in the absence of a severe clinical event is unlikely due to (a) variability of expression resulting in minimal clinical signs and symptoms (especially in women), (b) misattribution of SCD to other causes (e.g., accidents), (c) uninformative family structure (small family, mostly females), and (d) lack of local tertiary-level diagnostic testing $[9,10]$. Relying solely on cascade ge- netic screening may result in unidentified TMEM43 p.S358L carriers in the population. If this is indeed the case, PBGS for TMEM43 p.S358L may serve as an additional screening tool in this population where a high burden of disease coexists with lifesaving prophylactic treatment $[5-7,11]$.

PBGS has historically been used for preconception counseling in couples at risk of transmitting genetic disease as well as in the newborn period for identification of autosomal recessive conditions. However recently, research has focused on the utility of PBGS for carrier detection of autosomal dominant conditions which clinically present in early adulthood. These conditions include BRCA-related hereditary breast and ovarian cancer (HBOC), hereditary nonpolyposis colorectal cancer (HNPCC), and familial hypercholesterolemia (FH). As a group, these have been classified as Tier 1 genetic conditions by the Centers of Disease Control and Prevention (CDC), for which early identification and intervention have a meaningful clinical impact [12]. Many studies have supported the use of PBGS in high-risk populations BRCA1/2 screening for HBOC in Ashkenazi Jewish women [13-20], MLH1/MSH2/MSH6/PMS2 screening for HNPCC in women diagnosed with endometrial cancer [21], and APOB/LDLR/PCSK9 screening for FH in South Africans [22] as well as in the general population [23-25]. These studies also highlight that a significant number of newly identified carriers, some of which have a diagnosis of cancer related to their underlying genetic variant [25], do not meet clinical criteria for genetic screening based on local guidelines [13-15, 19, 21-23, 25]. Similar to the CDC Tier 1 genetic conditions, it is likely that many TMEM43 p.S358L carriers remain unidentified in the province due to the innate inadequacies of cascade genetic screening in conjunction with NL's founder population. The purpose of this study was to explore the acceptability of pathogenic variant-specific PBGS for TMEM43 p.S358L in NL.

\section{Materials and Methods}

\section{Study Design}

This was a prospective cohort study which assessed (1) the impact of PBGS for the TMEM43 p.S358L variant on psychological distress and quality of life (QOL) up to 1-year post-genetic testing and (2) public attitudes towards this method of detection.

\section{Study Population}

All individuals who had at least 1 grandparent from NL and who could provide informed consent were eligible. 


\section{Participant Recruitment}

The majority of study participants were recruited through advertisements on television, radio, and in community newspapers in geographical regions of the island of Newfoundland known to have families with ARVC. A small proportion of participants had heard about the study through a family member or friend and had contacted the Provincial Medical Genetics Program directly, while others had expressed their interest in participating while visiting the genetics clinic for an unrelated reason. Before determining eligibility, the coordinator (author CS) asked the potential participants (a) how did you hear about the study and (b) 2 closed-ended Likert survey items assessing their attitude towards population sampling. This included (1) on a scale of 1 (not) to 5 (very), how anxious were you after seeing the advertisement and (2) on a scale of 1 (not) to 5 (very), do you think identifying people with a potentially serious heart condition using an advertisement in a newspaper is a good idea. In this way, public attitudes were recorded even in those who chose to not take part in the study or were ineligible. For those eligible and willing, the coordinator collected contact information and mailed a study package, including an introduction letter and a consent form. Those ineligible or not wishing to continue were thanked for their time.

\section{Variant Testing}

A cardiac Genetic Counselor (GC) (author FC) contacted the participant within 2 weeks to review the consent form. Participants were then offered an appointment at which the GC provided in depth counseling about ARVC caused by TMEM43 p.S358L. This included an explanation of the possible results of the genetic test, implications for themselves and family members, and a discussion of psychosocial issues related to genetic screening. It was explained that any individual found to have the TMEM43 p.S358L variant would be referred to Eastern Health's Cardiac Genetic Clinic for management. All potential participants were informed that a copy of their DNA test result would be provided to their primary care physician. Following counseling, if the participant wished to continue, a spit sample from which DNA could be extracted was obtained. DNA was extracted from saliva as per recognized protocols $[26,27]$ and determination of the presence or absence of the TMEM43 p.S358L variant was assessed using Sanger sequencing [3].

\section{Quantitative Assessments}

Demographic information was obtained initially by the GC. Participants were asked to complete 2 surveys at baseline, 6 months, and 1 year to measure health-related QOL and psychological distress. Follow-up surveys were completed by mail. Validated measures included the SF-36v2 and the Impact of Events Scale (IES), both used extensively to assess psychological well-being across numerous health conditions.

The SF-36v2 contains 36 questions which assess health-related QOL. The sections of the SF-36v2 include physical functioning, role physical, bodily pain, general health, vitality, social functioning, role emotional, and mental health. Scaled scores were transformed to a $0-100$ scale; lower scores reflect higher levels of disability and higher scores reflect lower levels of disability [28].

The IES is a 15-item scale which assesses an individual's level of psychological distress in response to an identifiable event [29] - in this case, the event was defined as undergoing predic-

Population-Based Genetic Screening for ARVC

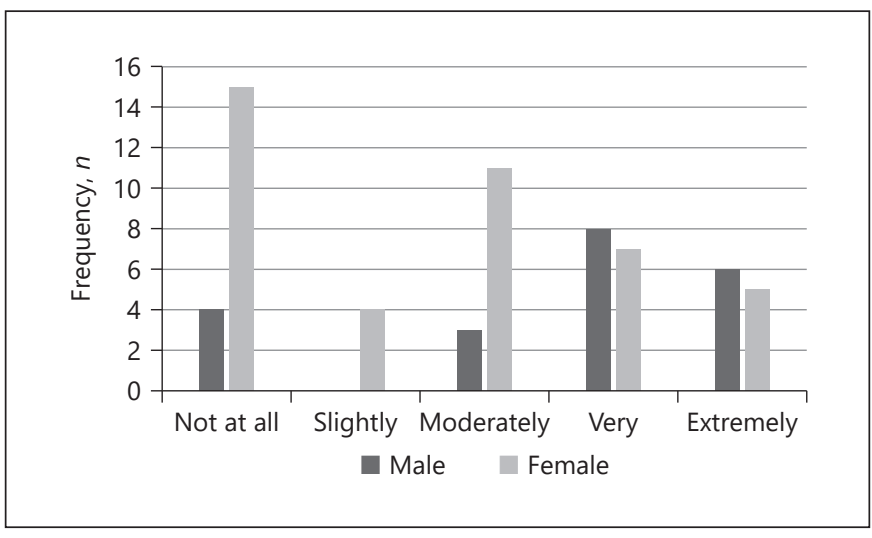

Fig. 1. Reported anxiety levels upon seeing or hearing advertisements designed to inform potential participants about the genetic screening study for TMEM43 p.S358L on television, radio, and/or in community newspapers by 63 individuals who inquired about the study ( 21 males, 42 females). No significant differences were noted.

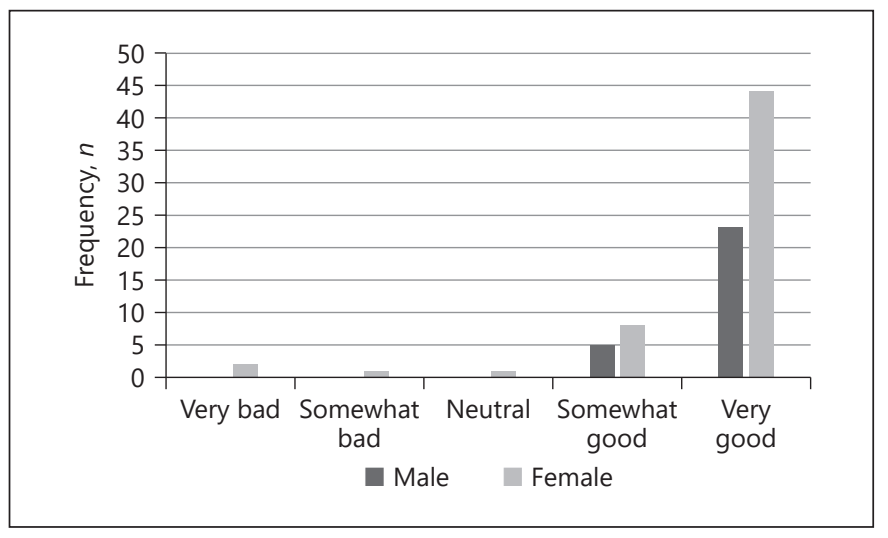

Fig. 2. Opinions on the use of advertisements on television, radio, and/or in community newspapers from 84 individuals $(28$ males and 56 females) who inquired about the genetic screening study for TMEM43 p.S358L. No significant differences were noted.

tive genetic testing for the TMEM43 p.S358L variant. Each question is scored on a Likert scale based on the participant's response and weighted between not at all $=0$ and often $=5$ [29]. The range of global scores falls between 0 and 75 [29]. Two subscales were created, including an Intrusive Subscale and an Avoidant Subscale which range from 0 to 35 and $0-40$, respectively [29]. The intrusive subscale identifies if an individual is experiencing intrusive thoughts, nightmares, feelings, or imagery associated with the event; the Avoidant Subscale identifies if an individual is actively avoiding thoughts and feelings associated with the event [29].

\section{Statistical Analysis}

Descriptive statistics, in the form of frequency (proportion) and mean \pm standard deviation, were conducted on categorical and continuous variables, respectively. $\chi^{2}$ analyses and logistic re- 
Table 1. Characteristics of the study population $(n=73)$

\begin{tabular}{lccc}
\hline & Male & Female & Total \\
\hline Sample size, $n(\%)$ & $23(31.5)$ & $50(68.5)$ & 73 \\
Age mean, years \pm SD & $50 \pm 14$ & $53 \pm 13$ & $52 \pm 13$ \\
Education, $n(\%)^{*}$ & & & \\
$\quad$ <High School & $1(4)$ & $5(10)$ & $6(8)$ \\
$\quad$ High school & $4(17)$ & $11(23)$ & $15(21)$ \\
$\quad$ College diploma & $13(57)$ & $18(38)$ & $31(44)$ \\
$\quad$ University degree & $5(22)$ & $14(29)$ & $19(27)$ \\
$\quad$ Missing data & 0 & 2 & 2 \\
Personal history of cardiac condition, $n(\%)$ & $4(17)$ & $12(24)$ & $16(22)$ \\
Family history of SCD, $n(\%)$ & $17(74)$ & $44(88)$ & $61(84)$ \\
Prior knowledge of ARVC, $n(\%)$ & $11(48)$ & $20(40)$ & $31(43)$ \\
History of genetic testing, $n(\%)$ & $2(9)$ & $3(6)$ & $5(7)$ \\
\hline
\end{tabular}

SCD, sudden cardiac death; ARVC, arrhythmogenic right ventricular cardiomyopathy. ${ }^{*}(\%)$ represents the valid percentage. gressions were used to identify categorical and continuous variables which were predictive of anxiety levels after seeing the study advertisement. Data from the SF-36v2 questionnaires were scored using the QualityMetric Health Outcomes Scoring Software Version 4.5. A repeated measures, linear mixed-effect model was performed to compare the means of the transformed scores for each component of the SF-36v2 and IES between the 3 time points (baseline, 6 months, and 1 year). The statistical software program SPSSv20 was used for all analyses and a $p$ value $\leq 0.05$ was considered statistically significant.

\section{Results}

\section{Demographic Data}

Ninety-four individuals inquired about the study, the majority of whom completed the 2 initial screening questions (Fig. 1, 2). Population-genetic screening for TMEM43 p.S358L was considered a somewhat $(15 \%)$ or a very good idea (80\%), with a minority (3\%) describing it as a poor method of screening. A degree of anxiety after seeing the advertisement was reported by $68 \%$ of participants, with $23 \%$ feeling very anxious and $17 \%$ feeling extremely anxious. Most respondents (58\%) felt moderate anxiety or less after seeing the advertisement. There were no significant differences between male and female respondents on opinions surrounding the advertisement or anxiety levels after seeing the advertisement.

Seventy-three individuals continued beyond the first phone call and provided a spit sample for testing. No TMEM43 p.S358L carriers were identified. DNA test results were provided to participants at a median of 3-month post sample being obtained. All but 1 participant received their genetic results in 6 months or less. Characteristics of the study population are in Table 1 . The mean age was 52 years, $69 \%(n=50)$ were female, and the majority had a post-secondary education (71\%). Through a self-report survey item, $84 \%$ indicated that a family member had passed away due to SCD. Extended family histories were fully explored with the GC during genetic counseling and 3 individuals were referred to the Eastern Health Cardiac Genetic Clinic for further evaluation, clinical screening, and follow-up. Thirty-one participants (43\%) stated they were familiar with ARVC primarily because there was an ARVC diagnosis within their extended family; in all cases, the affected family member was related via a distant marriage, and likely reflects the close-knit communities in these regions. Only 5 individuals (7\%) had undergone genetic testing in the past and had done so through other genetic research studies. There were no significant differences in baseline characteristics between male and female participants.

\section{Health-Related QOL: SF-36v2}

A repeated measures, linear mixed-effect model did not show any significant differences in the means of the transformed scores for each component of the SF-36v2 in participants who underwent genetic screening over the study period (Table 2).

\section{Psychological Distress Levels: IES}

The mean global and subscale scores for the IES were low and did not significantly change between baseline, 6 months, and 1 year using a repeated measures, linear mixed-effect model (Table 3 ). 
Table 2. SF-36v2 section scores $(0-100)$ at baseline, 6 months and 1 year post-genetic testing for TMEM43 p.S358L

\begin{tabular}{|c|c|c|c|c|c|c|c|}
\hline \multirow[t]{3}{*}{ SF-36v2 section } & \multicolumn{7}{|c|}{ Time of follow-up } \\
\hline & \multirow[t]{2}{*}{ baseline } & \multirow[t]{2}{*}{6 months } & \multirow[t]{2}{*}{1 year } & \multicolumn{2}{|c|}{ baseline to 6 months } & \multicolumn{2}{|c|}{ baseline to 1 year } \\
\hline & & & & $\mathrm{CI}$ & $p$ value & $\mathrm{CI}$ & $p$ value \\
\hline Physical functioning & $79.9 \pm 2.4$ & $81.9 \pm 2.8$ & $80.6 \pm 3.1$ & -6.9 to -2.9 & 0.420 & -6.1 to -4.8 & 0.802 \\
\hline Role physical & $84.1 \pm 2.6$ & $82.5 \pm 3.2$ & $83.3 \pm 3.4$ & -4.1 to -7.4 & 0.575 & -5.5 to -7.1 & 0.803 \\
\hline Bodily pain & $69.9 \pm 2.8$ & $69.5 \pm 3.2$ & $66.8 \pm 3.5$ & -5.1 to -6.0 & 0.868 & -3.0 to -9.3 & 0.308 \\
\hline General health & $66.9 \pm 2.4$ & $64.4 \pm 2.8$ & $64.5 \pm 3.0$ & -2.2 to -7.2 & 0.295 & -2.8 to -7.7 & 0.362 \\
\hline Vitality & $59.8 \pm 2.5$ & $58.5 \pm 3.0$ & $59.0 \pm 3.2$ & -3.7 to -6.4 & 0.602 & -4.8 to -6.5 & 0.767 \\
\hline Social functioning & $82.0 \pm 2.7$ & $80.0 \pm 3.2$ & $85.6 \pm 3.5$ & -3.8 to -8.2 & 0.462 & -10.2 to -3.1 & 0.289 \\
\hline Role emotional & $88.9 \pm 2.0$ & $87.6 \pm 2.5$ & $89.3 \pm 2.8$ & -3.6 to -6.2 & 0.594 & -5.8 to -5.0 & 0.881 \\
\hline Mental health & $77.3 \pm 2.5$ & $73.8 \pm 2.8$ & $77.0 \pm 3.0$ & -0.5 to -7.4 & 0.086 & -4.1 to -4.7 & 0.893 \\
\hline
\end{tabular}

Data presented as mean \pm standard error. Data analyzed using a repeated measures, linear mixed-effect model.

Table 3. IES section scores at baseline, 6 months, and 1 year post-genetic testing for TMEM43 p.S358L

\begin{tabular}{|c|c|c|c|c|c|c|c|}
\hline \multirow[t]{3}{*}{ IES section } & \multicolumn{7}{|c|}{ Time of follow-up } \\
\hline & \multirow[t]{2}{*}{ baseline } & \multirow[t]{2}{*}{6 months } & \multirow[t]{2}{*}{1 year } & \multicolumn{2}{|c|}{ baseline to 6 months } & \multicolumn{2}{|c|}{ baseline to 1 year } \\
\hline & & & & $\mathrm{CI}$ & $p$ value & $\mathrm{CI}$ & $p$ value \\
\hline Intrusive subscale range $0-35$ & $5.5 \pm 0.9$ & $4.9 \pm 1.2$ & $4.6 \pm 1.3$ & -1.8 to -3.0 & 0.635 & -1.7 to -3.6 & 0.493 \\
\hline Avoidant subscale range $0-40$ & $6.1 \pm 1.1$ & $5.4 \pm 1.3$ & $5.7 \pm 1.5$ & -1.9 to -3.2 & 0.604 & -2.5 to -3.3 & 0.780 \\
\hline Global subscale range $0-75$ & $11.0 \pm 1.9$ & $10.1 \pm 2.4$ & $9.6 \pm 2.7$ & -4.0 to -5.9 & 0.700 & -4.1 to -6.9 & 0.617 \\
\hline
\end{tabular}

Data presented as mean \pm standard error. Data analyzed using a repeated measures, linear mixed-effect model. IES, impact of event scale.

\section{Discussion}

This is the first study to assess public attitudes and the psychological impact of PBGS for the TMEM43 p.S358L pathogenic variant causing 1 form of ARVC. Despite some anxiety reported after seeing the study advertisement, we demonstrated public support for PBGS, consistent with other studies [16-20,24]. All participants tested negative for the TMEM43 p.S358L variant and did not experience any significant adverse psychological effect measured by the SF36-v2 and IES up to 1 year following genetic testing, a situation mirrored in previous PBGS studies for BRCA1/2 [16].

We did not identify any carriers of the TMEM43 p.S358L variant and cannot comment on the impact of being identified as a carrier via this method on psychological distress and QOL. It is likely that being identified as a carrier of TMEM43 p.S358L could affect QOL based on the previous research which highlights several areas of concern including employment restrictions, large financial burdens, and insurability post-genetic testing [30]. Furthermore, those screening positive for the TMEM43 p.S358L variant would have to choose whether to have placement of an ICD, which, although demonstrably lifesaving [5], is also known to impact psychological and physical well-being $[31,32]$. Young ICD patients with genetic forms of heart disease (including ARVC) have reported increased levels of depression, anxiety, and posttraumatic stress, and show decreased scores on QOL measures in the short-term [33-35]. Additional distress may also be caused by the burden of the risk of transmission to subsequent generations. The increase in survival of $>30$ years in males provided with an ICD for prophylaxis, $>20$ years in males with an ICD following clinical ventricular tachyarrhythmia's, and $>2$ years in females has likely allowed psychological sequalae to this disease 
to be recognized; historically, early SCD secondary to lethal arrhythmias or progressive cardiac dysfunction was the overwhelming impact on families and individuals.

The study sample is small, and we do not know if findings generalize to a wider population. Referrals to the Genetic Cardiac Clinic have increased for TMEM43 p.S358L testing (pers comm, FC), and anecdotally the demand from the public appears to be very high. This is reflected in the number of people who responded to this research project who declared a family history of SCD. Perhaps unsurprisingly, there was significant loss to follow-up in our study population of noncarriers, potentially creating a selection bias in those respondents who completed follow-up surveys. Nonetheless, we showed it was acceptable to provide PBGS for the TMEM43 p.S358L variant and demonstrated that this form of screening is perceived favorably by the public despite some degree of anxiety.

Newfoundland's founder population makes it an ideal location for PBGS not only for ARVC caused by TMEM43 p.S358L but also for other hereditary forms of cardiac disease with high prevalence. Although the aim of our study was to assess the impact of PBGS for TMEM43 p.S358L on psychological health and QOL, our findings beg the question of whether PBGS for TMEM43 p.S358L should be implemented in NL to identify at risk carriers. As elucidated in the 1960s, the features which make a disease suitable for population screening include a disease with a high prevalence in a recognized population, the ability to identify asymptomatic carriers through noninvasive testing, and the availability of treatments which, when provided early, improve prognosis [11] While most screening programs to date utilize noninvasive tests such as pap smears, mammograms, and fecal immunochemistry to identify at risk individuals, there has been a push toward offering genetic-based population screening for several hereditary conditions. Of these, the CDC has recognized HBOC, HNPCC, and FH as Tier 1 genetic conditions for which early identification and intervention results in a clinically significant outcome [12]. ARVC caused by TMEM43 p.S358L is a classic example of a CDC Tier 1 genetic condition - a disease which affects a distinct population that can be identified through genetic screening and has a proven impact on survival when treatment is offered [12]. The discovery of TMEM43 p.S358L in NL has also led the American College of Medical Genetics and Genomics (ACMG) to classify TMEM43 as one of the "ACMG 59" - genes where specific variants are known to cause disorders with clinical phenotypes that are serious, and where clinical action is available and acceptable [36]. Detecting p.S358L in the TMEM43 gene by exome or ge- nome sequencing is now recognized as an actionable finding as disease outcome is modified substantially by early, presymptomatic treatment with an ICD [36].

In conclusion, we show acceptance of PBGS among research subjects who screened negative for the TMEM43 p.S358L variant in NL with minimal impact on anxiety levels, health-related QOL, and psychological distress. Although we were not able to identify any new carriers due to a small sample size, we believe that PBGS for the TMEM43 p.S358L variant could serve as an additional screening tool in NL to detect carriers who would benefit from lifesaving treatment with an ICD. Our local infrastructure, which includes the Cardiac Genetics Program and Electrophysiology Department, makes PBGS for this pathogenic variant potentially feasible.

\section{Acknowledgements}

We acknowledge with gratitude the families who support our research, our funders, and the SCD clinical and research team at Memorial University.

\section{Statement of Ethics}

This research was conducted in accordance with the Tri-Council Policy Statement: Ethical Conduct for Research Involving $\mathrm{Hu}-$ mans - TCPS 2 and the World Medical Association Declaration of Helsinki. All participants have given their written informed consent. The Health Research Ethics Board (HREB) of NL (File Ref \# 12.138) approved this study.

\section{Conflict of Interest Statement}

The authors wish to declare that they have none to disclose.

\section{Funding Sources}

This research was supported by grants from Genome Canada (Atlantic Medical Genetic and Genomics Initiative); the Atlantic Canada Opportunities Agency - Atlantic Innovation Fund; the Research Development Corporation; the Translational and Personalized Medicine Initiative (funded by the Canadian Institutes of Health Research, the Government of NL, and IBM); the Faculty of Medicine, Memorial University; and St. Jude Medical.

\section{Author Contributions}

Cassidy Brothers and Kathleen Hodgkinson contributed equally to the manuscript: Kathleen Hodgkinson and Holly Etchegary were co-supervisors for Cassidy Brothers research project. Terry- 
Lynn Young (molecular genetics), Daryl Pullman (bioethics and $\mathrm{GE}^{3} \mathrm{LS}$ ), and Sean Connors (clinical electrophysiology) were all leads for the SCD research project and involved in the manuscript preparation. Fiona Curtis was the GC. Charlene Simmonds was the Research Manager/Coordinator/Assistant and oversaw data collection and storage. Jim Houston (RA) completed genetic testing for the TMEM43 mutation from participant samples in TerryLynn Young's laboratory. Hensley H. Mariathas helped with statistical analysis.

\section{Data Availability Statement}

All data generated or analyzed for the study are available upon request to the corresponding author.

\section{References}

1 Basso C, Corrado D, Marcus FI, Nava A, Thiene G. Arrhythmogenic right ventricular cardiomyopathy. Lancet. 2009;373(9671): 1289-300.

2 Corrado D, Thiene G. Arrhythmogenic right ventricular cardiomyopathy/dysplasia: clinical impact of molecular genetic studies. Circulation. 2006;113(13):1634-7.

3 Merner ND, Hodgkinson KA, Haywood AF, Connors S, French VM, Drenckhahn JD, et al. Arrhythmogenic right ventricular cardiomyopathy type 5 (ARVD5) is a fully penetrant, lethal arrhythmic disorder caused by a missense variant in the TMEM43 gene. Am J HumGenet. 2008;82(4):809-21.

4 Hodgkinson KA, Connors S, Merner N, Haywood A, Young TL, McKenna WJ, et al. The natural history of a genetic subtype of arrhythmogenic right ventricular cardiomyopathy caused by a p.S358L variant in TMEM43. Clinical Genetics. 2013;83(4):321-31.

5 Hodgkinson KA, Howes AJ, Boland P, Shen XS, Stuckless S, Young TL, et al. Long-term clinical outcome of arrhythmogenic right ventricular cardiomyopathy in individuals with a p.S358L variant in TMEM43 following implantable cardioverter defibrillator therapy. Circ Arrhythm Electrophysiol. 2016;9(3): e003589.

6 Paulin FL, Hodgkinson KA, MacLaughlan S, Stuckless SN, Templeton C, Shah S, et al. Exercise and arrhythmic risk in TMEM43 p.S358L arrhythmogenic right ventricular cardiomyopathy. Heart Rhythm. 2020;17(7): 1159-66.

7 Milting H, Klauke B, Christensen AH, Müsebeck J, Walhorn V, Grannemann S, et al. The TMEM43 Newfoundland mutation p.S358L causing ARVC-5 was imported from Europe and increases the stiffness of the cell nucleus. Eur Heart J. 2015;36(14):872-81.

8 Morris JK. Is cascade testing a sensible method of population screening? J Med Screen. 2004;11(2):57-8.

9 Green RC, McLaughlin JR, Younghusband HB. SISE matters: the sum of information on seventy-year-old equivalents measures pedigree information content when assessing the risk of HNPCC in a family. Fam Cancer. 2005; 4(2):169-75.
10 McKenna WJ, Thiene G, Nava A, BlomstromLundqvist C, Fontaine G, Camerini F. Diagnosis of arrhythmogenic right ventricular dysplasia/cardiomyopathy. Task force of the working group myocardial and pericardial disease of the european society of cardiology and the scientific council on cardiomyopathies of the international society and federation of cardiology. Br Heart J. 1994;71(3): 215-8.

11 Wilson J, Junger G. Principles and practice of screening for disease. France: WHO; 1968.

12 Centers for Disease Control and Prevention, Office of Public Health Genomics. Tier 1 genomic applications toolkit for public health departments; 2019. Available from: https:// www.cdc.gov/genomics/implementation/ toolkit/index.htm.

13 Manchanda R, Loggenberg K, Sanderson S, Burnell M, Wardle J, Gessler V, et al. Population testing for cancer predisposing BRCA1/ BRCA2 variants in the Ashkenazi-Jewish community: a randomized controlled trial. J Natl Canc Inst. 2015;107(1):379.

14 Metcalfe KA, Poll A, Royer R, Nanda S, Llacuachaqui $\mathrm{M}$, Sun $\mathrm{P}$, et al. A comparison of the detection of BRCA mutation carriers through the provision of Jewish population-based genetic testing compared with clinic-based genetic testing. Br J Cancer. 2013;109(3):777-9.

15 Metcalfe KA, Poll A, Royer R, Llacuachaqui M, Tulman A, Sun P, et al. Screening for founder mutations in BRCA1 and BRCA2 in unselected Jewish women. J Clin Oncol. 2010; 28(3):387-91.

16 Metcalfe KA, Poll A, Llacuachaqui M, Nanda S, Tulman A, Mian N, et al. Patient satisfaction and cancer-related distress among unselected Jewish women undergoing genetic testing for BRCA1 and BRCA2. Clin Genet. 2010;78(5):411-7.

17 Metcalfe KA, Mian N, Enmore M, Poll A, Llacuachaqui $M$, Nanda $S$, et al. Long-term follow-up of Jewish women with a BRCA1 and BRCA2 mutation who underwent population genetic screening. Breast Cancer Res Treat. 2012;133(2):735-40.

18 Cousens N, Kaur R, Meiser B, Andrews L. Community attitudes towards a Jewish community BRCA1/2 testing program. Fam Cancer. 2017;16(1):17-28.
19 Lieberman S, Tomer A, Ben-Chetrit A, Olsha O, Strano S, Beeri R, et al. Population screening for BRCA1/BRCA2 founder variants in Ashkenazi Jews: proactive recruitment compared with self-referral. Genet Med. 2016; 19(7):754-62.

20 Shkedi-Rafid S, Gabai-Kapara E, GrinshpunCohen J, Levy-Lahad E. BRCA genetic testing of individuals from families with low prevalence of cancer: experiences of carriers and implications for population screening. Genet Med. 2012;14(7):688-94.

21 Chao X, Li L, Wu M, Ma S, Tan X, Zhong S, et al. Comparison of screening strategies for Lynch syndrome in patients with newly diagnosed endometrial cancer: a prospective cohort study in China. Cancer Commun. 2019; 39(1):42.

22 Kotze MJ, Peeters AV, Loubser O, Theart L, du Plessis L, Hayes VM, et al. Familial hypercholesterolemia: potential diagnostic value of mutation screening in a pediatric population of South Africa. Clin Genet. 1998;54(1):74-8.

23 Rowley SM, Mascarenhas L, Devereux L, Li N, Amarasinghe KC, Zethoven M, et al. Population-based genetic testing of asymptomatic women for breast and ovarian cancer susceptibility. Genet Med. 2019;21(4):913.

24 Akbari MR, Gojska N, Narod SA. Coming of age in Canada: a study of population-based genetic testing for breast and ovarian cancer. Curr Oncol. 2017;24(5):282.

25 Grzymski JJ, Elhanan G, Morales Rosado JA, Smith E, Schlauch KA, Read R, et al. Population genetic screening efficiently identifies carriers of autosomal dominant diseases. Nat Med. 2020;26(8):1235-9.

26 Koressaar T, Remm M. Enhancements and modifications of primer design program Primer3. Bioinformatics. 2007;23(10):1289-91.

27 Untergasser A, Cutcutache I, Koressaar T, Ye J, Faircloth BC, Remm M, et al. Primer3 new capabilities and interfaces. Nucleic Acids Res. 2012;40(15):e115.

28 Maruish M, DeRosa M. A guide to the integration of certified short form survery scoring and data quality evaluation capabilities. Lincoln: QualityMetric Incorporated; 2009.

29 Horowitz M, Wilner N, Alvarez W. Impact of event scale: a measure of subjective stress. Psychosom Med. 1979;41(3):209-18. 
30 Etchegary H, Enright G, Audas R, Pullman D, Young TL, Hodgkinson K. Perceived economic burden associated with an inherited cardiac condition: a qualitative inquiry with families affected by arrhythmogenic right ventricular cardiomyopathy. Genet Med. 2015;18(6):584-92.

31 Etchegary H, Pullman D, Connors SP, Simmonds C, Young TL, Hodgkinson KA. "There are days I wish it wasn't there, and there's days I realize I'm lucky": a qualitative study of psychological sequelae to the implantable cardioverter defibrillator as a treatment for the prevention of sudden cardiac death in arrhythmogenic right ventricular cardiomyopathy. J R Soc Med. 2017;18(6):1-9.
32 Pullman D, Hodgkinson K. The curious case of the De-ICD: negotiating the dynamics of autonomy and paternalism in complex clinical relationships. Am J Bioeth. 2016;16(8):310.

33 Ingles J, Sarina T, Kasparian N, Semsarian C. Psychological wellbeing and posttraumatic stress associated with implantable cardioverter defibrillator therapy in young adults with genetic heart disease. Int J Cardiol. 2013; 168(4):3779-84.
34 Verkerk AJ, Vermeer AM, Smets EM, Dekker LR, Wilde AA, Van Langen IM, et al. Quality of life in young adult patients with a cardiogenetic condition receiving an ICD for primary prevention of sudden cardiac death. Pacing Clin Electrophysiol. 2015;38(7):870-7.

35 Webster G, Panek KA, Labella M, Taylor GA, Gauvreau K, Cecchin F, et al. Psychiatric functioning and quality of life in young patients with cardiac rhythm devices. Pediatrics. 2014;133(4):e964-72.

36 Green RC, Berg JS, Grody WW, Kalia SS, Korf BR, Martin CL, et al. ACMG recommendations for reporting of incidental findings in clinical exome and genome sequencing. Genet Med. 2013;15(7):565-74. 